\title{
ASSEMBLEIA HOLOCÊNICA DE VERTEBRADOS DE PEQUENO PORTE DO SÍTIO ALCOBAÇA, ESTADO DE PERNAMBUCO, BRASIL
}

\author{
DARIVAL FERREIRA, ANA KAROLINE BARROS SILVA, \\ CLÁUDIA HELENA CYSNEROS MATOS \\ Unidade Acadêmica de Serra Talhada, UFRPE, Fazenda Saco, s/n, Cx.P. 063, Serra Talhada, PE, Brasil. \\ darival.fds@gmail.com, karolbarros.biologia@gmail.com, c.helena@uast.ufrpe.br \\ PATRÍCIA HADLER \\ Departamento de Geociências, CFCH, UFSC, Campus Universitário, s/n, Florianópolis, SC, Brasil. \\ patricia.hadler@ufsc.br \\ ANNIE SCHMALTZ HSIOU \\ Departamento de Biologia, FFCLRP, USP, Av. Bandeirantes 3900, Ribeirão Preto, SP, Brasil. \\ anniehsiou@ffclrp.usp.br
}

\begin{abstract}
A HOLOCENE ASSEMBLAGE OF SMALL VERTEBRATES FROM ALCOBAÇA SITE, PERNAMBUCO STATE, BRAZIL. The record of small vertebrates from the Holocene of northeast of Brazil is very scarce, being restricted to archaeological studies. The studied material consists of cranial and mandibular fragments of lizards, marsupials, and caviomorph rodents excavated from Alcobaça site, Buíque County, Pernambuco State. The site has radiocarbon dates ranging from 4,851 \pm 30 years BP to $888 \pm 25$ years BP. Kerodon rupestris (Wied, 1820), Galea sp. (Caviidae), Thrichomys sp., Phyllomys sp. (Echimyidae), Monodelphis sp. (Didelphidae), Tropidurus sp. (Tropiduridae), Tupinambis sp., and "Ameiva" sp. (Teiidae) were recorded. The Phyllomys sp. remains constitute the first record of this taxon for the Quaternary of northeastern Brazil. The fauna suggests a paleoenvironment very similar to the current one, characterized as "brejo de altitude".
\end{abstract}

Key words: Squamata, Didelphimorphia, Caviomorpha, Holocene, paleoenvironment, Parque Nacional do Catimbau.

RESUMO - O registro de pequenos vertebrados para o Holoceno da região Nordeste do Brasil é bastante escasso, ficando restrito a trabalhos de cunho arqueológico. O material estudado constitui-se de fragmentos cranianos e mandibulares de lagartos, mamíferos marsupiais e roedores caviomorfos provenientes do sítio Alcobaça, localizado no Parque Nacional do Catimbau, município de Buíque, Estado de Pernambuco. O sítio possui datações radiocarbônicas abrangendo desde $4.851 \pm 30$ anos AP até $888 \pm 25$ anos AP. Registrou-se Tropidurus sp. (Tropiduridae), Tupinambis sp., “Ameiva” sp. (Teiidae), Monodelphis sp. (Didelphidae), Kerodon rupestris (Wied, 1820), Galea sp. (Caviidae), Thrichomys sp. e Phyllomys sp. (Echimyidae), sendo esse último registrado pela primeira vez para o Quaternário do nordeste do Brasil. Essa fauna sugere um paleoambiente muito similar ao atual, caracterizado como "brejo de altitude".

Palavras-chave: Squamata, Didelphimorphia, Caviomorpha, Holoceno, paleoambiente, Parque Nacional do Catimbau.

\section{INTRODUÇÃO}

O Estado de Pernambuco apresenta grande quantidade de depósitos fossilíferos quaternários, porém os trabalhos concentram-se principalmente nos registros da megafauna pleistocênica provenientes de tanques, formações típicas do Nordeste brasileiro (Silva et al., 2006; Oliveira et al., 2009). A fossilização de pequenos vertebrados nos tanques é rara (Araújo-Júnior \& Porpino, 2011) e o estudo dos pequenos vertebrados holocênicos está restrito a citações em trabalhos arqueológicos (e.g. Nascimento, 2001; De Queiroz $\&$ De Carvalho, 2008). No entanto, os grupos de pequenos vertebrados constituem-se em excelentes indicadores paleoambientais por possuírem necessidades ecológicas mais específicas (Rodrigues, 2008; Camolez \& Zaher, 2010). Além disso, a abundância no registro arqueológico de espécies ainda viventes permite um maior refinamento paleoambiental para o Holoceno (Perez, 2009).

Este trabalho tem por objetivo descrever a fauna de pequenos vertebrados (Squamata, Didelphimorphia e Rodentia) provenientes do sítio Alcobaça.

\section{ÁREA DE ESTUDO}

O sítio Alcobaça localiza-se no Município de Buíque, na área do Parque Nacional do Catimbau, situado na região central do Estado de Pernambuco, sob as coordenadas $8^{\circ} 32^{\prime} 24^{\prime \prime} \mathrm{S}, 37^{\circ} 11^{\prime} 39^{\prime \prime} \mathrm{O}$ (Figura 1). Trata-se de um abrigo 
sob rocha de constituição arenítica, com uma área de aproximadamente $980 \mathrm{~m}^{2}$, localizado na meia encosta de uma serra e próximo a um corpo de água permanente. $\mathrm{O}$ ambiente circunvizinho ao sítio é caracterizado como um "brejo de altitude" (Nascimento, 2001).

O Parque Nacional do Catimbau possui uma extensão de mais de 62.000 ha e está inserido na Bacia Hidrográfica do Rio Moxotó (SNE, 2002). Aárea abrange parte dos municípios de Buíque, Ibimirim e Tupanatinga, situado na transição entre o agreste e o sertão (IBAMA, 2008). O clima é semiárido com temperatura anual média de $26^{\circ} \mathrm{C}$ e precipitação média em torno de 450 a $950 \mathrm{~mm}$, sendo as chuvas bastante irregulares (Lôbo, 2008). O Parque Nacional do Catimbau situa-se no domínio da Caatinga, com uma heterogeneidade de espécies e estruturas (Lôbo, 2008), sendo encontrados na área elementos do cerrado, campos rupestres, mata atlântica e restinga (IBAMA, 2008). O domínio das Caatingas é comprovadamente a região cuja biodiversidade é a menos conhecida da América do Sul, no entanto com alto índice de diversidade (Vital et al., 2008). O Parque Nacional do Catimbau é considerado uma área de extrema importância apresentar números relevantes de endemismos e espécies raras (Vital et al., 2008; Geise et al., 2010). Na base de algumas das serras do Parque Nacional do Catimbau, formam-se vales, regiões isoladas de vegetação úmida em meio à vegetação típica da Caatinga circundante, conhecida no Nordeste como "brejo de altitude", os quais possuem uma biota típica, com uma flora formada por um mosaico de espécies comuns à Mata Atlântica e Amazônica (SNE, 2002; Sousa et al., 2004). Os "brejos de altitude" são verdadeiros enclaves de floresta úmida no semi-árido por receberem maior precipitação anual do que as regiões circunvizinhas.

A fauna atual do Parque Nacional do Catimbau é representada por uma diversidade de espécies. Entre os répteis, 30 espécies foram registradas, sendo os lagartos representados pelas famílias Iguanidae, Tropiduridae, Phyllodactylidae, Gekkonidae, Sphaerodactylidae, Teiidae, Gymnophthalmidae, Scincidae, Leiosauridae e Anguidae (Muniz \& Santos, 2009). Em relação aos mamíferos terrestres de pequeno porte, Geise et al. (2010) reportaram Didelphidae, roedores Cricetidae, Caviidae e Echimyidae.

Os achados arqueológicos incluem material lítico, como
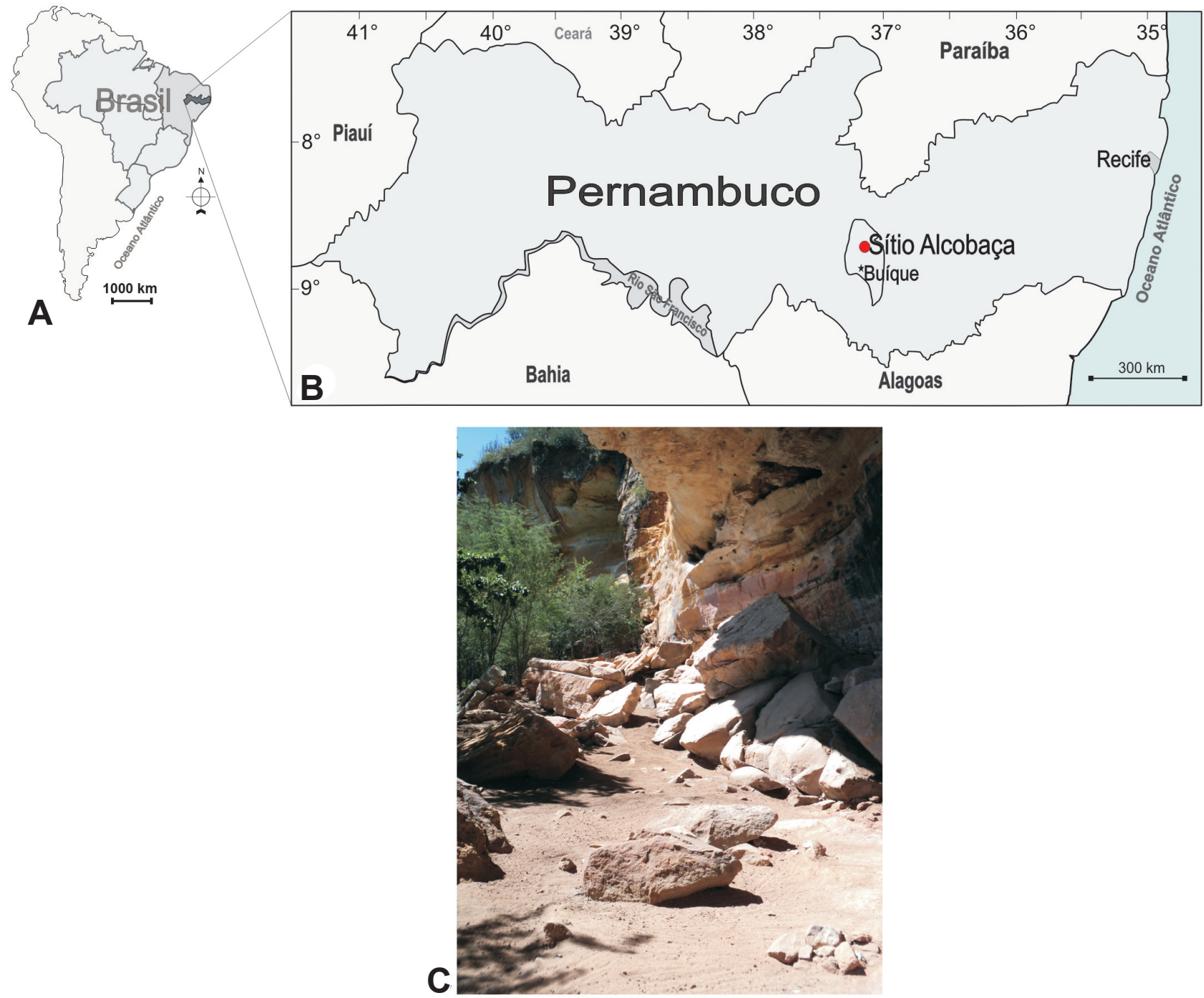

Figura 1. A-B, Localização geográfica do sítio Alcobaça; C, vista geral do sítio Alcobaça.

Figure 1. A-B, Geographic location of Alcobaça site; C, overview of the Alcobaça site. 
almofariz, raspador, lâmina e furador, além de cerâmica e pinturas rupestres (Nascimento, 2001). O material foi atribuído à Tradição Agreste, que tem um caráter nômade, habitando uma grande área de captação, mas dependente da demanda de recursos, especialmente água (Proença, 2008). Neste sítio foram encontrados restos faunísticos, onde marcas de fogo e de corte sugerem a utilização desses animais como alimentação (De Queiroz \& De Carvalho, 2008). São conhecidas 24 datações para o sítio que abrangem desde 4.851 \pm 30 anos AP até $888 \pm 25$ anos AP (Nascimento, 2001).

\section{MATERIAL E MÉTODOS}

O material pertence ao Núcleo de Estudos Arqueológicos da Universidade Federal de Pernambuco (NEA-UFPE) e constitui-se de 113 fragmentos crânio-mandibulares de pequenos vertebrados, com ou sem dentes inclusos, provenientes das quadrículas 203F e 204E do sítio Alcobaça. O material foi separado do sedimento através de peneiramento manual e limpo, sob estereomicroscópio, com pincel, água e agulha histológica. O material foi armazenado em tubos plásticos do tipo "eppendorf" ou em tubos criogênicos, dependendo do tamanho, numerado e posteriormente estudado sob estereomicroscópio. A identificação se baseou na comparação com material recente da Coleção de Mastozoologia da Unidade Acadêmica de Serra Talhada da Universidade Federal Rural de Pernambuco e com fotografias de material recente e fóssil, além de descrições e ilustrações da literatura. A sistemática segue Wilson \& Reeder (2005) para mamíferos e Estes et al. (1988), Frost \& Etheridge (1989), Frost et al. (2001a), Nydam \& Cifelli (2002) e Nydam et al. (2007) para lagartos. Na descrição dos lagartos seguiuse Presch (1970) e Estes et al. (1988) e na dos marsupiais Oliveira \& Goin (2006). Na nomenclatura das estruturas ósseas de Rodentia seguiu-se Woods \& Howland (1979) e nas dentárias seguiu-se Contreras (1964) e Emmons \& Vucetich (1998).

\section{SISTEMÁTICA PALEONTOLÓGICA}

\author{
Ordem SQUAMATA Oppel, 1811 \\ Subordem IGUANIA Cope, 1864 \\ Família TROPIDURIDAE Frost \& Etheridge 1989 sensu \\ Frost, Janies \& Titus, 2001a
}

Tropidurus Wied-Neuwied, 1825

Tropidurus sp.

(Figuras 2A-D)

Material estudado. NEA 1453-1, fragmento de maxilar esquerdo; NEA 1857-2, fragmento de maxilar esquerdo; NEA 2376-2, hemimandíbula direita completa.

Procedência estratigráfica. NEA 1453-1, Quadrícula 203-F, Camada 2, Nível 2/2; NEA 1857-2, Quadrícula 203-F, Camada 2, Nível 5; NEA 2376-2, Setor Área II, nível limpeza.

Comentários. Os exemplares atribuídos a Tropidurus sp. apresentam características peculiares ao gênero, como a superfície labial dos maxilares sem ornamentações e dentição heterodonte, formada por dentes anteriores robustos e unicuspidados, enquanto que os posteriores são tricuspidados (ver Camolez \& Zaher, 2010, fig. 48A). O porte é pequeno em relação à Tupinambis sp. e as cúspides dos dentes são muito proeminentes, em relação aos exemplares também descritos neste trabalho de "Ameiva" ameiva e Tupinambis sp. Os forames labiais são grandes, paralelos e apresentamse crescentes ântero-posteriormente. A hemimandíbula é delicada, rasa e comparativamente mais baixa do que o espécime de Tupinambis ou qualquer outro exemplar da família Teiidae, com dentário bem alongado (Brizuela, 2010). O canal de Meckel encontra-se fechado por toda sua extensão. A atribuição específica não foi possível pela escassez de material e de exemplares comparativos.

Tropidurus é um gênero distribuído por todo o continente sul-americano (Rodrigues, 1987; Frost et al., 2001b) e tem uma dieta diversificada sujeita a variações sazonais (Arruda $e t$ al., 2007; Ribeiro, 2010). Para o Parque Nacional do Catimbau foram registradas três espécies do gênero: Tropidurus hispidus (Spix, 1825), T. cocorobensis (Rodrigues, 1987) e T. semitaeniatus (Spix, 1825) (Muniz \& Santos, 2009). Para o Quaternário do Brasil, Camolez \& Zaher (2010) relataram a ocorrência de Tropidurus sp. para os estados da Bahia e Minas Gerais.

\section{Subordem SCLEROGLOSSA Estes, de Queiroz \& Gauthier, 1988 \\ Infra-ordem SCINCOMORPHA Camp, 1923 Família TEIIDAE Gray, 1827 \\ Subfamília TUPINAMBINAE Presch, 1974}

Tupinambis Daudin, 1802

Tupinambis sp.

(Figuras 2E-I)

Material. NEA 1857-1, hemimandíbula esquerda incompleta; NEA 1857-3, hemimandíbula direita incompleta.

Procedência estratigráfica. Quadrícula 203-F, Camada 2, Nível 5.

Comentários. As características diagnósticas para identificação do gênero foram: o grande porte do material, o dentário robusto e o tipo de substituição dentária (subpleurodonte) (Estes et al., 1988), a plataforma subdental que forma um sulco no dentário, onde ocorre a implantação dentária, a morfologia dos dentes molariformes que possuem base alargada com intensa deposição de cimento e a superfície oclusal estriada (Hsiou, 2007; Camolez \& Zaher, 2010). Não foi possível a identificação ao nível de espécie, pela fragmentação do material e pelo padrão conservativo da osteologia destes animais (ver Camolez, 2006).

A distribuição geográfica de Tupinambis abrange toda a América do Sul, dos Andes até a Patagônia (Ávila-Pires, 1995). A alimentação é basicamente onívora (Vanzolini et al., 1980). Para a herpetofauna do Parque Nacional do Catimbau, 
Muniz \& Santos (2009) registraram T. merianae (Duméril \& Bibron, 1839). Para o gênero há registro para o Quaternário do Brasil para os estados da Bahia, Goiás, Minas Gerais e Rio Grande do Sul (Hsiou, 2007; Camolez \& Zaher, 2010).

Subfamília TEIINAE Presch, 1974

“Ameiva” Meyer, 1795

"Ameiva" sp.

(Figuras 2J-K)

Material estudado. NEA 2376-1, hemimandíbula direita incompleta.

Procedência estratigráfica. Setor Área II, nível limpeza. Comentários. No espécime estudado o esplenial apresenta dois forames, sendo o maior deles o forame alveolar inferior disposto posteriormente, e o menor, o forame milohioide anterior, disposto anteriormente. A distribuição desses forames permitiu a atribuição à subfamília Teiinae, a qual inclui os gêneros "Ameiva", Kentropyx, "Cnemidophorus", Teius, Aspidoscelis e Dicrodon (Brizuela, 2010). Dentre as características diagnósticas observadas para o gênero "Ameiva" estão: o grande porte do exemplar, sendo maior do que grande parte dos táxons atuais da subfamília Teiinae, como "Cnemidophorus" (Wagler, 1830) e Kentropyx (Spix, 1825); presença de dentário marcadamente côncavo; dentição heterodonte, sendo os anteriores unicuspidados e os posteriores mais robustos e bi- ou tricuspidados e implantação dos dentes do tipo subpleurodonte, com a presença de alvéolos de substituição (Presch, 1970; Brizuela, 2010; Camolez \& Zaher, 2010). A escassez de material e a ausência de elementos diagnósticos não permitiram a atribuição específica do material.

A distribuição geográfica do gênero "Ameiva" é ampla na América do Sul, ocorrendo desde o norte do Panamá até o leste dos Andes (Vitt et al., 2008). No Brasil está presente em grande parte do território, principalmente em matas ensolaradas e áreas de vegetação aberta (Vanzolini, 1972). Apresenta estratégia alimentar do tipo forrageamento ativo (Huey \& Pianka, 1981), com amplo espectro alimentar, predominando em sua dieta pequenos artrópodes (Martins, 1991; Vitt \& Colli, 1994). Para o Quaternário do Brasil, "Ameiva" ameiva foi registrada para os Estados de Goiás, Bahia e Minas Gerais (Camolez \& Zaher, 2010).

Classe MAMMALIA Linnaeus, 1758

Ordem DIDELPHIMORPHIA Gill, 1872

Família DIDELPHIDAE Gray, 1821

Material. NEA 1816-1, fragmento de dentário direito com c e p2; NEA 1894-1, fragmento de dentário direito edéntulo; NEA 1990-1, fragmento de dentário direito edéntulo; NEA 2163-2, fragmento de dentário direito edéntulo.

Procedência estratigráfica. NEA 1816-1, Quadrícula 203-F, Setor II, Camada 2, Nível 5; NEA1894-1, Quadrícula 203-F, Camada 2, Nível 5/2; NEA2163-2, Quadrícula 203-F, Setor ANAI, Camada 2, Nível 5/7; NEA 1990-1, Quadrícula 203-F, Camada 2, Nível 5.
Comentários. O material está muito fragmentado e em sua grande maioria sem os dentes preservados, o que dificultou a identificação a nível genérico. No espécime com os dentes preservados, NEA 1816-1, os mesmos estão muito desgastados, não sendo possível a observação de estruturas diagnósticas. Dessa maneira, optou-se por deixar os espécimes acima mencionados identificados apenas ao nível de família.

Monodelphis Burnett, 1830

Monodelphis sp.

(Figura 3)

Material. NEA 2393-1, fragmento de maxilar esquerdo com C-M4; NEA 2163-1, fragmento de maxilar direito com P3-M4.

Procedência estratigráfica. NEA 2393-1, Quadrícula 204-E, Setor II, Camada 2, Nível-3; NEA 2163-1, Quadrícula 203-F, setor ANA I, Camada 2, Nível 5/7.

Comentários. O material apresenta características diagnósticas para o gênero, como ausência da cúspide estilar $\mathrm{C}$ e um metacone vestigial no M4 (Goin \& Rey, 1997; Hadler et al., 2009a). O grande número de espécies para o gênero, a ausência de claras características distintivas ao nível de espécie, o pequeno número de espécimes encontrados e o desgaste dos dentes dificultaram uma identificação mais precisa.

A distribuição geográfica do gênero Monodelphis é ampla e abrange toda a América do Sul, podendo ser encontrado desde florestas úmidas e densas até as mais secas, entretanto não tolera grandes altitudes (Eisenberg \& Redford, 1999). O seu hábito é generalista, com uma dieta muito variada, sendo comumente terrestre e noturno (Emmons, 1997; Oliveira, 2010). Entre a mastofauna atual do Parque Nacional do Catimbau, Geise et al. (2010) registraram duas espécies de marsupiais: Gracilinanus agilis (Burmeister, 1854) e Monodelphis domestica (Wagner, 1842). Esta última é a espécie vivente mais abundantemente reportada para a Caatinga (Oliveira et al., 2003). Monodelphis já foi registrado para o Quaternário dos Estados de São Paulo, Rio Grande do Sul, Pará, Goiás, Mato Grosso do Sul, Ceará e Minas Gerais (Hadler et al., 2009b e referências contidas; Oliveira, 2010).

Ordem RODENTIA Bowdich, 1821 Subordem HYSTRICOMORPHA Brandt, 1855 Infra-ordem HYSTRICOGNATHI Brandt, 1855 Família CAVIIDAE Fischer de Waldheim, 1817

Subfamília CAVIINAE Fischer de Waldheim, 1817

Galea Meyen, 1833

Galea sp.

(Figuras 4A-B)

Material. NEA 1182-1, fragmento de dentário esquerdo edêntulo; NEA 1258-1, maxilar esquerdo com P4-M3; NEA 1258-2, fragmento de dentário direito edêntulo; NEA 1453-1, fragmento de dentário direito com p4-m2; NEA 


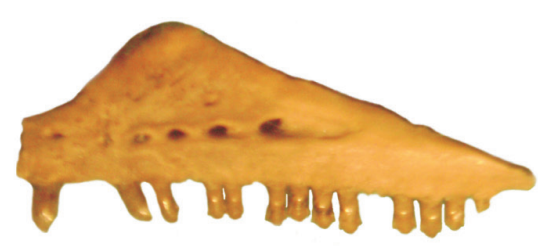

A
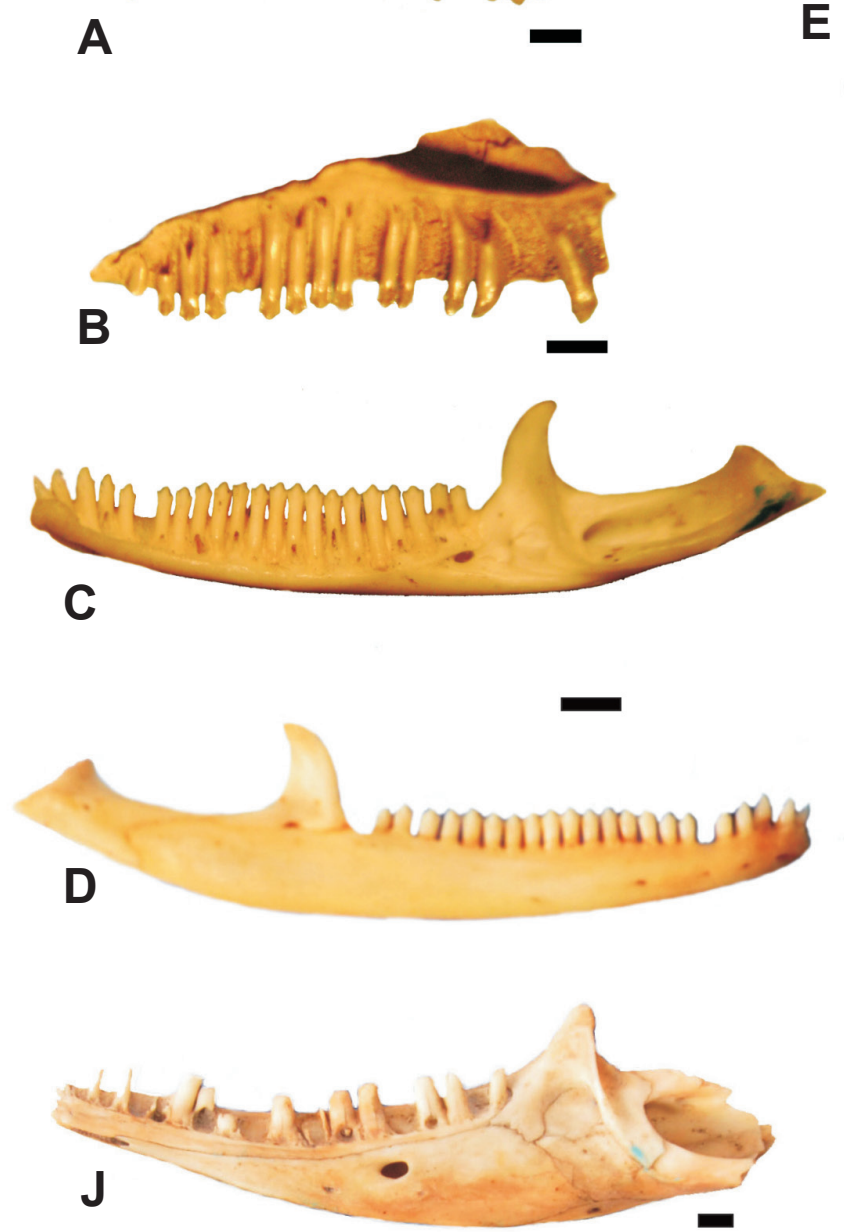

$\mathbf{E}$
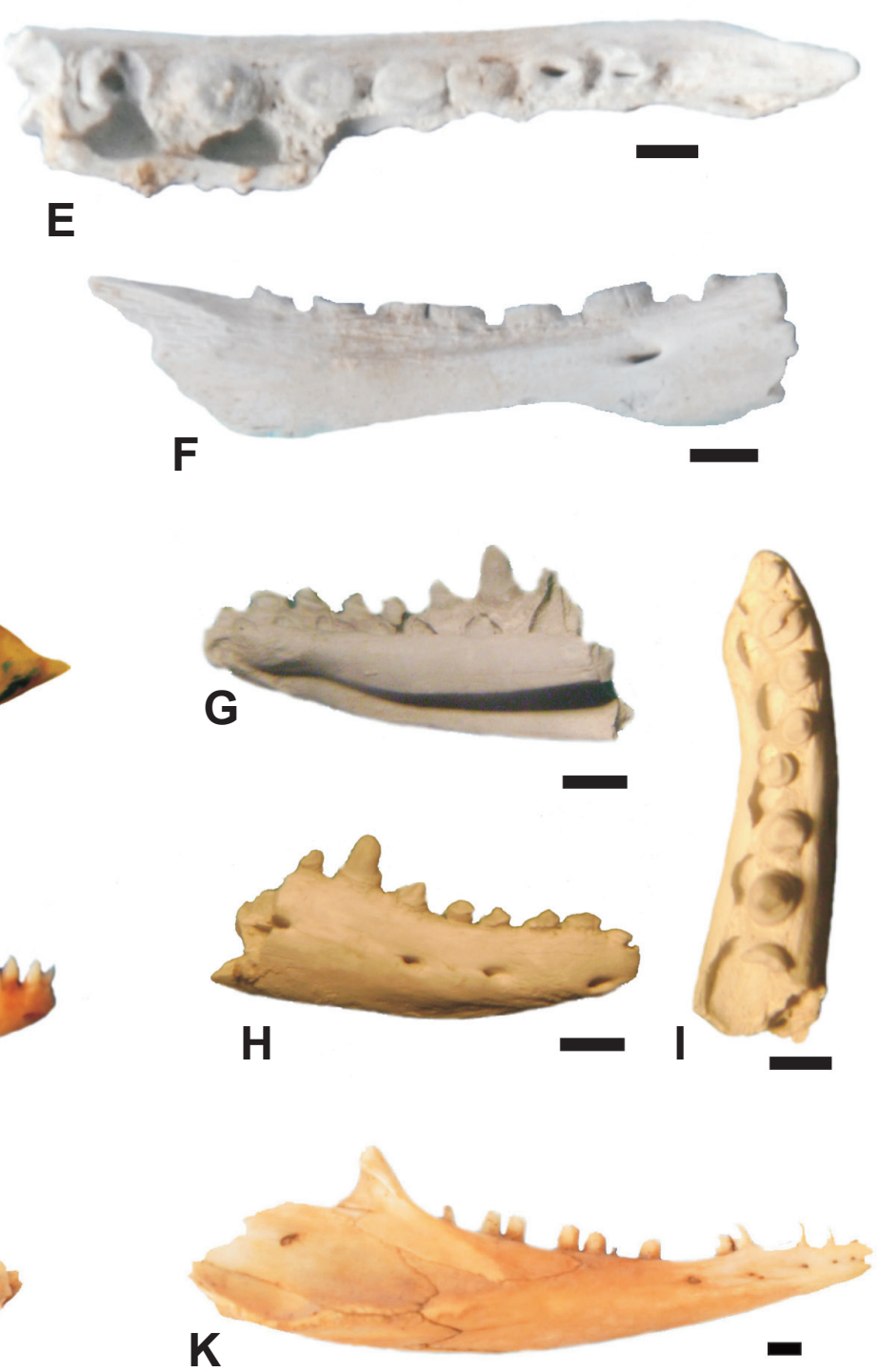

Figura 2. Restos fósseis de Squamata do sítio Alcobaça. A-D, Tropidurus sp. A-B, NEA 1453-1 fragmento de maxilar esquerdo, A, vista lateral; B, vista medial; C-D, NEA 2376-2, hemimandíbula direita completa, C, vista medial; D, vista lateral. E-I, Tupinambis sp. E-F, NEA 1857-1, hemimandíbula esquerda incompleta, E, vista oclusal; F, vista lateral; G-I, NEA 1857-3, hemimandíbula direita incompleta, G, vista medial; H, vista lateral; I, vista oclusal. J-K "Ameiva" sp. NEA 2376-1, hemimandíbula direita incompleta, J, vista medial; K, vista lateral. Escalas = 1mm.

Figure 2. Squamata fossil remains from the Alcobaça site. A-D, Tropidurus sp. A-B, NEA 1453-1, fragment of left maxilla, A, lateral view; B, medial view; C-D, NEA 2376-2, right complete hemimandible, C, medial view; D, lateal view. E-I, Tupinambis sp. E-F, NEA 1857-1, left incomplete hemimandible, E, occlusal view; F, lateral view; G-I, NEA 1857-3, right incomplete hemimandible, G, medial view; H, lateral view; I, occlusal view. J-K, "Ameiva" sp. NEA 2376-1, right incomplete hemimandible, J, medial view; L, lateral view. Scale bars $=1 \mathrm{~mm}$.

1620-1, fragmento de dentário esquerdo edêntulo; NEA 1816-1, fragmento de dentário direito com m1; NEA 18574, fragmento de dentário esquerdo com p4; NEA 1857-5, fragmento de dentário esquerdo edêntulo; NEA 1857-7, fragmento de dentário direito edêntulo; NEA 1857-13, fragmento de dentário direito edêntulo; NEA 1857-23, fragmento de dentário direito edêntulo; NEA 1894-2, fragmento de maxilar direito edêntulo; NEA 1918-4, dentário direito com m1; NEA 1918-5, fragmento de dentário esquerdo com p4-m2; NEA 1918-7, fragmento de dentário direito com p4; NEA 1918-9, fragmento de dentário esquerdo edêntulo; NEA 1918-11, fragmento de dentário direito com p4; NEA 1918-12, fragmento de dentário direito edêntulo; NEA 191816, fragmento de maxilar direito edêntulo; NEA 1990-1, maxilar esquerdo edêntulo; NEA 1990-2, maxilar esquerdo edêntulo; NEA 1990-4, maxilar direito edêntulo; NEA 1990-6, maxilar esquerdo edêntulo; NEA 1990-7, maxilar esquerdo edêntulo; NEA 1990-11, fragmento de dentário esquerdo com m2-m3; NEA 1990-18, fragmento de dentário direito com p4-m1; NEA 1990-19, fragmento de dentário esquerdo com m1; NEA 1990-24, fragmento de dentário esquerdo edêntulo; NEA 1990-27, fragmento de dentário direito edêntulo; NEA 2002-3, dentário direito edêntulo; NEA 2053-1, dentário direito com m1-m3; NEA 2294-1, maxilar direito com P4M2; NEA 2294-4, fragmento de dentário direito edêntulo; NEA 2298-2, dentário esquerdo com p4-m3; NEA 2321-1, fragmento de dentário direito com p4-m1; NEA 2321-2, dentário direito com p4-m3. 


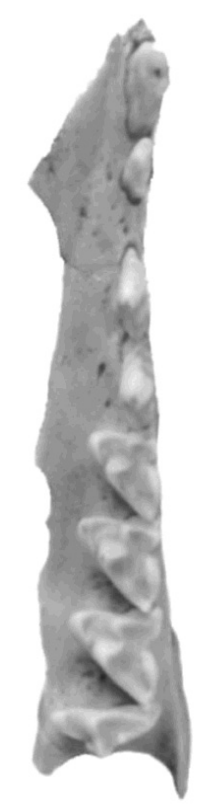

A

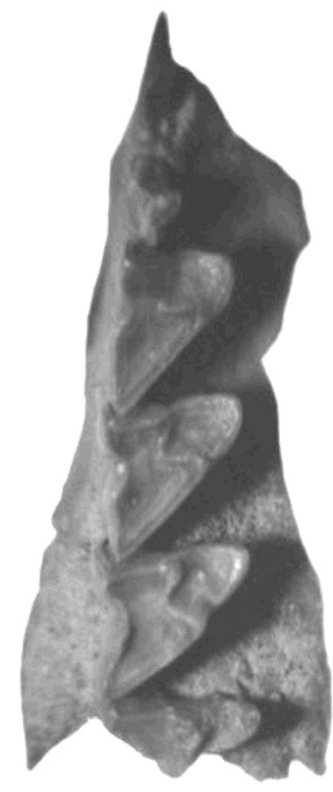

B
Figura 3. Monodelphis sp. A, NEA 2393-1, fragmento de maxilar esquerdo com C-M4 em vista oclusal; B, NEA 2163-1, fragmento de maxilar direito com P3-M4 em vista oclusal. Escala $=1 \mathrm{~mm}$.

Figure 3. Monodelphis sp. A, NEA 2393-1, fragment of left maxilla with C-M4 in occlusal view; B, NEA 2163-1, fragment of right maxilla with P3-M4 in occlusal view. Scale bar $=1 \mathrm{~mm}$.

Procedência estratigráfica. NEA 1182-1, Quadrícula 203F, Camada 2, Nível 1; NEA 1258-1, NEA 1258-2, Quadrícula 203F, Camada 2, Nível 2; NEA 1453-1, Quadrícula 203F, Camada 2, Nível 2/2; NEA 1620-1, Quadrícula 203F, Camada 2, Nível 3; NEA 1857-4, NEA 1857-5, NEA 1857-7, NEA 1857-13, NEA 1857-23, NEA 1816-1, Quadrícula 203F, Camada 2, Nível 5; NEA 1894-2, NEA 1918-4, NEA 19185, NEA 1918-7, NEA 1918-9, NEA 1918-11, NEA 1918-12, NEA 1918-16, Quadrícula 203F, Camada 2, Nível 5/2; NEA 1990-1, NEA 1990-2, NEA 1990-4, NEA 1990-6, NEA 19907, NEA 1990-11, NEA 1990-18, NEA 1990-19, NEA 1990-24, NEA 1990-27, NEA 2002-3, Quadrícula 203F, Camada 2, Nível 5/4; NEA 2053-1, Quadrícula 203F, Camada 2, Nível 5/5; NEA 2294-1, NEA 2294-4, NEA 2298-2, Quadrícula 204 E, Camada 2, Nível 2; NEA 2321-1, NEA 2321-2, Quadrícula 204 E, Camada 2, Nível 3.

Comentários. Segundo Kerber et al. (2011), as mandíbulas do gênero Galea podem ser identificadas através do início da crista horizontal ao nível do prisma I do p4, da porção anterior da fossa massetérica profunda e presença de cemento no hipofléxido, características observadas nos exemplares estudados. Além disso, os espécimes de Galea apresentam uma tuberosidade massetérica mais ampla e desenvolvida quando comparada com Kerodon (também presente nesta localidade, vide abaixo). A fossa maxilar é curta e profunda. A crista massetérica inicia ao nível do $\mathrm{p} 4$, característica compartilhada pelas espécies atuais do gênero (Ubilla, 1996).
Nos espécimes estudados há a presença de apenas uma fenda em um dos prismas, assim como observado por Ubilla (1996) e Kerber et al. (2011). No prisma I do M3 há o desenvolvimento de uma fenda externa e no prisma II há uma leve fenda terciária, o que não é relatado no trabalho de Ubilla (1996). Não há interrupção do esmalte na série p4-m3, de maneira contrária ao observado nos espécimes atuais de G. musteloides Meyen, 1832, onde é constatada essa interrupção na face interna de ambos os prismas (Ubilla, 1996). A taxonomia ainda incerta do gênero associada à variação intraespecífica (Contreras, 1964) e a necessidade de comparação com um número maior de espécimes recentes do gênero, levaram à uma identificação apenas genérica do material.

Galea é um gênero que está distribuído pelo Brasil, Paraguai, Bolívia, Argentina, Peru e Chile (Bezerra, 2008). Adaptado a ambientes abertos, habitando no Brasil principalmente os biomas Caatinga e Cerrado, sua principal fonte de alimentação são as gramíneas (Eisenberg \& Redford, 1999). Nos levantamentos faunísticos do Parque Nacional do Catimbau é registrada a ocorrência de G. spixii (Wagler, 1831) (SNE, 2002; Geise et al., 2010). Para o Quaternário brasileiro há registros deste gênero para os Estados de Piauí, Sergipe, Minas Gerais e Rio Grande do Sul (Winge, 1888; Guérin et al., 1993; Dantas, 2009; Kerber et al., 2011).

\section{Subfamília HYDROCHOERINAE Gray, 1825}

Kerodon F. Cuvier, 1825

Kerodon rupestris (Wied, 1820)

(Figuras 4C-D)

Material. NEA 1258-3, fragmento de maxilar esquerdo edêntulo; NEA 1857-21, fragmento de dentário esquerdo edêntulo; NEA 1918-1, maxilar direito com P4-M1; NEA 1918-6, fragmento de dentário direito edêntulo; NEA 19188, fragmento de dentário direito edêntulo; NEA 1918-14, fragmento de dentário esquerdo edêntulo; NEA 1990-3, fragmento de maxilar direito edêntulo; NEA 1990-12, fragmento de dentário direito edêntulo; NEA 1990-16, fragmento de dentário direito com m2-m3; NEA 1990-17, fragmento de dentário esquerdo com p4-m1; NEA 199020, fragmento de dentário direito edêntulo; NEA 1990-21, fragmento de maxilar esquerdo com P4; NEA 1990-26, fragmento de dentário direito com m1; NEA 1990-31, fragmento de maxilar direito com P4-M1; NEA 2032-2, fragmento de dentário direito com p4- m1; NEA 21961, fragmento de dentário direito edêntulo; NEA 2196-3, fragmento de dentário direito; NEA 2196-5, fragmento de dentário esquerdo edêntulo.

Procedência estratigráfica. NEA 1258-3, Quadrícula 203F, Camada 2, Nível 2; NEA 1857-21, Quadrícula 203F, Camada 2, Nível 5; NEA 1918-1, NEA 1918-6, NEA 1918-8, NEA 1918-14, Quadrícula 203F, Camada 2, Nível 5/2; NEA 1990-3, NEA 1990-12, NEA 1990-16, NEA 1990-17, NEA 1990-20, NEA 1990-21, NEA 1990-26, NEA 1990-31, NEA 2032-2, Quadrícula 203F, Camada 2, Nível 5/4; NEA 2196-1, NEA 
2196-3, NEA 2196-5, Quadrícula 203F, Camada 2, Nível 5/9.

Comentários. O prisma anterior do $\mathrm{P} 4$ menor do que o posterior e o $\mathrm{p} 4$ triprismático permitiram atribuir os espécimes a esse gênero. A tuberosidade massetérica é plana e pouco desenvolvida quando comparada aos espécimes de Galea. A porção anterior do arco zigomático apresenta-se estreita e o diastema é bastante longo em relação a Galea, com sua margem ventral formando um ângulo agudo com a base da mandíbula. A fossa maxilar é rasa e ampla. A atribuição específica se deu pela semelhança com os espécimes recentes e também pelo argumento de Lessa et al. (2005) de que Kerodon acrobata Moojen, Locks \& Langguth, 1997 surgiu de processos ecológicos ou históricos recentes, sendo este provavelmente um produto da especiação de $K$. rupestris.

Kerodon é um gênero exclusivamente brasileiro, e a espécie K. rupestris é endêmica da Caatinga (Oliveira et al., 2003). Ela habita os afloramentos rochosos da região semiárida, com vegetação arbustiva rasteira, alimentando-se de sementes e folhas da vegetação que crescem em seu território (Alho, 1982; Eisenberg \& Redford, 1999). Está registrada para o Parque Nacional do Catimbau (SNE, 2002). O gênero Kerodon já foi registrado para o Quaternário dos Estados da Bahia, Ceará, Piaú (Guérin, 1991; Lessa et al., 2005; Lessa \& Pessoa, 2005; Oliveira, 2010).

Família ECHIMYIDAE Gray, 1825 Subfamília ECHIMYINAE Gray, 1825

Phyllomys Lund, 1839

Phyllomys sp.

(Figura 4E)

Material. NEA 1857-1, fragmento de maxilar esquerdo com P4 e M1; NEA 1857-25, dente molar esquerdo (?).

Procedência estratigráfica. Quadrícula 203F, Camada 2, Nível 5.

Comentários. O gênero Phyllomys é similar ao gênero Diplomys pelos dentes retangulares e formados por quatro lofos paralelos. No entanto, Phyllomys apresenta uma ligação entre os lofos, diferentemente de Diplomys Thomas, 1916 (Leite, 2003; Emmons, 2005). O material aqui estudado demonstra grande semelhança com o gênero Phyllomys, especialmente quando comparado com material analisado por Hadler et al. (2008). Devido ao pequeno número de espécimes e ao caráter fragmentário da amostra, a identificação ao nível de espécie não foi possível.

Phyllomys é conhecido como rato de espinho, equimiídeo arborícola endêmico da Mata Atlântica e que se alimenta principalmente de folhas (Leite, 2003). Sua distribuição ocorre por todo litoral brasileiro, e também no interior de alguns estados como, Ceará, Pernambuco, Bahia e Minas Gerais (Emmons et al., 2002; Leite, 2003; Oliveira et al., 2003). Até o presente momento não foi registrado entre a fauna atual do Parque Nacional do Catimbau. Há registro para o Quaternário de Minas Gerais, São Paulo e Rio Grande do Sul (Lund, 1840; Hadler et al., 2008; Castro \& Langer, 2011).
Subfamília EUMYSOPINAE Rusconi, 1935

Thrichomys Trouessart, 1880

Thrichomys sp.

(Figuras 4F-G)

Material. NEA 1453-2, fragmento de dentário direito com m2; NEA 1453-3, fragmento de dentário esquerdo edêntulo; NEA 1857-2, fragmento de dentário direito com m1-m3; NEA 1857-6, dentário direito edêntulo; NEA 1857-8, dentário esquerdo com m2-m3; NEA 1857-9, dentário esquerdo edêntulo; NEA 1857-10, fragmento de dentário direito com m1-m3; NEA 1857-11, fragmento de dentário esquerdo edêntulo; NEA 1857-14, fragmento de dentário esquerdo edêntulo; NEA 1857-15, fragmento de dentário esquerdo edêntulo; NEA 1857-16, fragmento de dentário esquerdo com m1-m2; NEA 1857-17, fragmento de dentário direito com p4-m1; NEA 1857-18, fragmento de dentário direito com m1; NEA 1857-19, fragmento de dentário esquerdo edêntulo; NEA 1857-20, fragmento de dentário esquerdo com m2- m3; NEA 1918-2, palato com ambos P4-M3; NEA 19185, dentário esquerdo com p4- m3; NEA 1918-10, fragmento de dentário direito com p4; NEA 1918-13, dentário direito com p4-m2; NEA 1918-15, fragmento de dentário esquerdo com p4-m2; NEA 1990-5, fragmento de maxilar direito com P4-M2; NEA 1990-8, fragmento de maxilar esquerdo com P4-M1; NEA 1990-9, maxilar esquerdo com P4-M1; NEA 1990-10, dentário direito com m1-m2; NEA 1990-13, dentário direito com p4-m3; NEA 1990-14, fragmento de dentário esquerdo com p4-m2; NEA 1990-15, dentário direito com m1- m3; NEA 1990-22, fragmento de dentário direito com m1-m2; NEA 1990-23, fragmento de dentário esquerdo com m1; NEA 1990-25, fragmento de dentário direito com p4-m2; NEA 1990-28, fragmento de dentário esquerdo com p4-m1; NEA 1990-29, fragmento de dentário esquerdo com m1; NEA 1990-30 fragmento de dentário esquerdo com m2-m3; NEA 1990-32, fragmento de dentário direito com m2; NEA 1990-33, fragmento de dentário direito com p4; NEA 2002-2, dentário esquerdo com p4-m3; NEA 2032-1, dentário esquerdo com p4-m3; NEA 2053-2, dentário direito com p4-m3; NEA 2196-1, palato com ambos P4-M3; NEA 2294-2, fragmento de dentário esquerdo com m1-m2; NEA 2294-3, dentário direito com p4-m3; NEA 2376-2, fragmento de dentário direito com m1-m3; NEA 2391-1, fragmento de dentário direito com $\mathrm{p} 4-\mathrm{m} 3$.

Procedência estratigráfica. NEA 1857-2, NEA 1857-8, NEA 1857-6, NEA 1857-9, NEA 1857-10, NEA 1857-11, NEA 1857-14, NEA 1857-15, NEA 1857-16, NEA 1857-17, NEA 1857-18, NEA 1857-19, NEA 1857-20, Quadrícula 203F, Camada 2, Nível 2; NEA 1453-2, NEA 1453-3, Quadrícula 203F, Camada 2, Nível 2/2; NEA 1918-2, NEA 1918-5, NEA 1918-10, NEA 1918-13, NEA 1918-15, Quadrícula 203F, Camada 2, Nível 5/2; NEA 1990-5, NEA 1990-8, NEA 199013, NEA 1990-14, NEA 1990-15, NEA 1990-22, NEA 1990-9, NEA 1990-10, NEA 1990-25, NEA 1990-23, NEA 1990-28, NEA 1990-29, NEA 1990-30, NEA 1990-32, NEA 1990-33, 

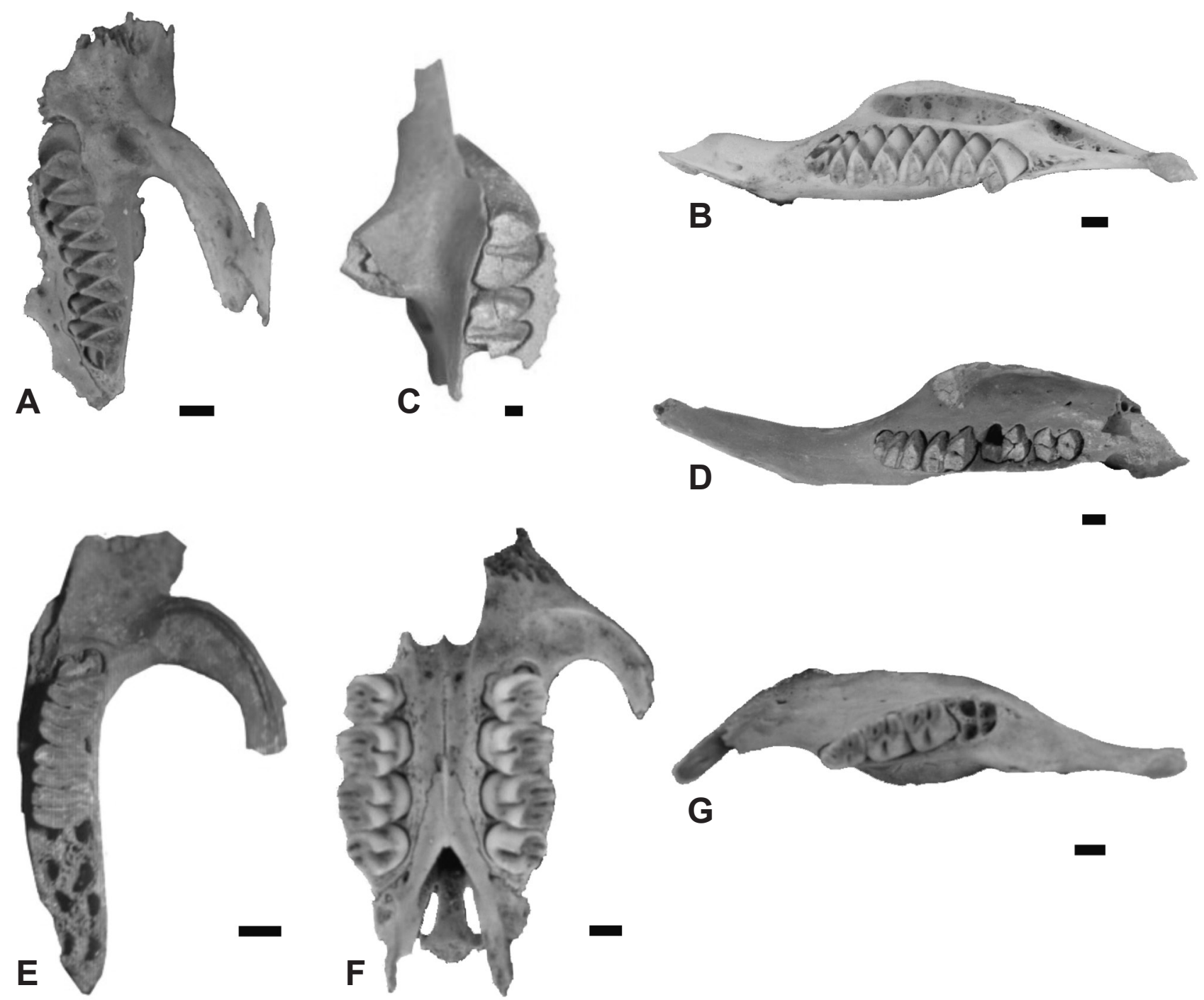

Figura 4. A-B, Galea sp. A, NEA 1258-1, maxilar esquerdo com P4-M3 em vista oclusal; B, NEA2321-2, dentário direito com p4-m3 em vista oclusal. C-D, Kerodon rupestris, C, NEA 1990-31, fragmento de maxilar direito com P4-M1 em vista oclusal; D, NEA 2032-2, fragmento de dentário direito com p4-m1 em vista oclusal. E, Phyllomys sp. NEA 1857-1, fragmento de maxilar esquerdo com P4-M1 em vista oclusal. F-G, Thrichomys sp. F, NEA 2196-1, palato com P4-M3 em vista oclusal; G, NEA 1990-14, fragmento de dentário esquerdo com p4-m2 em vista oclusal. Escalas = 1mm.

Figure 4. A-B, Galea sp. A, NEA 1258-1, left maxilla with P4-M3 in occlusal view; B, NEA 2321-2, right dentary with p4-m3 in occlusal view. C-D, Kerodon rupestris, C, NEA 1990-31, fragment of right maxilla with P4-M1 in occlusal view; D, NEA 2032-2, fragment of right dentary with p4-m1 in occlusal view. E, Phyllomys sp. NEA 1857-1, fragment of left maxilla with P4-M1 in occlusal view. F-G, Thrichomys sp. F, NEA 2196-1, palate with P4-M3 in occlusal view; G, NEA 1990-14, fragment of left dentary with p4-m2 in occlusal view. Scale bars $=1 \mathrm{~mm}$.

NEA 2002-2, NEA 2032-1, Quadrícula 203F, Camada 2, Nível 5/4; NEA 2053-2, Quadrícula 203F, Camada 2, Nível 5/5; NEA 2196-1, Quadrícula 203F, Camada 2, Nível 5/9; NEA 2294-2, NEA 2294-3, NEA 2391-1, Quadrícula E-204, Nível 2; NEA 2376-2, limpeza.

Comentários. Os espécimes aqui estudados quando comparados com material recente do gênero Thrichomys e com dados de bibliografia (e.g. Emmons, 2005) demonstraram grande semelhança com esse táxon. Segundo Emmons (2005), o formato do anterolófido no p4, em dentes com pouco desgaste, é uma característica distintiva entre os táxons da família Echimyidae. Ainda segundo a autora, Carterodon sulcidens (Lund, 1841) e T. apereoides (Lund, 1839) possuem um anterolófido com formato não triangular, o que pode ser observado nos espécimes aqui estudados.
No entanto, $C$. sulcidens e $T$. apereoides diferem quanto à localização do forame mandibular, sendo em Thrichomys mais próximo do ramo, conforme observado nos espécimes estudados. Estudos recentes reconheceram quatro espécies para o gênero Thrichomys: T. apereoides, T. inermis Pictet, 1841, T. pachyurus (Wagner, 1845) e T. laurentius Thomas, 1904 (Neves \& Pessôa, 2011). O material analisado não apresenta características distintivas para reconhecimento ao nível específico.

Thrichomys é encontrado desde afloramentos rochosos até áreas abertas e florestais do Cerrado, Caatinga e Pantanal e possui hábito terrestre (Neves \& Pessôa, 2011). Thrichomys apereoides e T. laurentis são as espécies que atualmente ocorrem no estado de Pernambuco (Bonvicino et al., 2008; Neves \& Pessôa, 2011), sendo T. apereoides a única espécie, 
até então, registrada no Parque Nacional do Catimbau (SNE, 2002). Para o Quaternário, o gênero está registrado para os estados de Mato Grosso do Sul, Ceará, Piauí e Goiás (Guérin, 1991; Salles et al., 2006; Oliveira, 2010).

\section{CONSIDERAÇÕES FINAIS}

De modo geral, o material está em bom estado de preservação, apesar de fragmentado. $\mathrm{Na}$ assembleia do sítio Alcobaça, foram registrados lagartos das famílias Tropiduridae (Tropidurus sp.) e Teiidae ("Ameiva" sp. e Tupinambis sp.), marsupiais da família Didelphidae (Monodelphis sp.) e roedores caviomorfos das famílias Caviidae (Kerodon rupestris e Galea sp.) e Echimyidae (Thrichomys sp.e Phyllomys sp.), sendo Phyllomys registrado pela primeira vez para o Quaternário do Nordeste brasileiro.

Nos níveis analisados observa-se uma maior quantidade de espécimes de Kerodon rupestris, Galea sp. e Thrichomys sp., enquanto os demais táxons estão menos representados. O grande número de espécimes de roedores caviomorfos em oposição ao de marsupiais e lagartos, pode estar refletindo questões tafonômicas. De Queiroz \& De Carvalho (2008) identificaram marcas de predação humana em pequenos vertebrados do sítio Alcobaça, principalmente em mamíferos, ao contrário do que é observado em restos coletados em sítios arqueológicos da região Sul e Norte do país.

Para o Brasil, os registros de mamíferos holocênicos são pontuais estando localizados principalmente em Minas Gerais, Bahia, São Paulo e Rio Grande do Sul (Hadler et al., 2009b e referências; Castro \& Langer, 2011). No entanto, muitas dessas localidades não possuem datações, e as que existem, muitas vezes, indicam mistura temporal, o que prejudica a inferência paleoambiental em escala regional (Auler et al., 2006). A herpetofauna quaternária possui um registro ainda mais pontual, sendo os escamados de um modo geral registrados em seis estados brasileiros: Bahia, Ceará, Minas Gerais, São Paulo, Rio Grande do Sul e Goiás (Estes, 1983; Hsiou, 2007; Hsiou et al., 2009; Camolez \& Zaher 2010; Oliveira et al., 2011). Nesse sentido destacam-se os trabalhos em sítios arqueológicos, como o Alcobaça, com grande número de datações.

A assembleia holocênica de pequenos vertebrados não difere de maneira significativa daquela atualmente encontrada no Parque Nacional do Catimbau por Muniz \& Santos (2009) e Geise et al. (2010), com exceção de Phyllomys sp., o qual não foi registrado para a localidade até o momento. Em relação ao paleoambiente, os táxons de lagartos e marsupiais resultam pouco informativos pela sua escassez, enquanto os roedores caviomorfos, remetem à vegetação de Caatinga, a qual predomina nos dias atuais na área. Táxons típicos da Caatinga, como Kerodon rupestris, Galea sp. e outros bem adaptados a essa vegetação como Thrichomys, são os predominantes. Phyllomys sp., característico da Mata Atlântica, pode indicar a ocorrência de maior umidade e influência de elementos arbóreos de Mata Atlântica, refletindo as condições de "brejo de altitude", no entanto poucos exemplares desse táxon foram coletados. Os dados palinológicos indicam a predominância de vegetação de Caatinga no Nordeste brasileiro desde 42.000 anos AP, sendo que em alguns momentos foram registrados períodos de maior umidade, como por exemplo, entre 15.500 e 11.800 anos AP, favorecendo a expansão das florestas (Behling et al., 2000). É provável que os "brejos de altitude", localizados na Caatinga em áreas úmidas, tenham surgido da retração dessas florestas. Lôbo (2008) estudou a palinologia do Parque Nacional do Catimbau e estabeleceu três ecozonas: Ecozona I, de 8.400 anos até 5.970 anos AP; Ecozona II, de 5.970 a 1.694 anos AP e a última, Ecozona III, de 1.694 anos $\mathrm{AP}$ até o Presente. Apenas as duas últimas têm correlação cronológica com o material do sítio Alcobaça. Para a Ecozona II, Lôbo (2008) sugeriu um ambiente mais diversificado do que o atual, especialmente em relação aos táxons arbóreos, o que seria justificado por uma maior umidade e a Ecozona III já teria as condições semelhantes às atuais. Essas mudanças no clima, especialmente quanto à umidade, acabaram não ficando registradas na fauna de pequenos vertebrados já que não há diferenças na composição taxonômica ao longo do tempo no sítio. Os dados palinológicos também indicam o início da interferência humana na vegetação ocorrendo na Ecozona II, o que coincide com os dados arqueológicos (Nascimento, 2001; Lôbo, 2008). Indicações de maior umidade na Caatinga durante o Holoceno (9.000 anos AP) também foram fornecidas por dados paleoparasitológicos obtidos através de ovos de Trichuris Roederer, 1761 em coprólitos de Kerodon rupestris (Araújo et al., 1993). Dessa maneira, o registro dos táxons de vertebrados de pequeno porte permite ampliar o conhecimento sobre sua distribuição temporal e geográfica e sobre o Quaternário do Estado de Pernambuco. Além disso, fornece dados para um melhor entendimento futuro da formação da Caatinga, bioma de ocorrência exclusiva no Brasil.

\section{AGRADECIMENTOS}

Os autores agradecem ao Núcleo de Estudos Arqueológicos da UFPE e à A.C. Ramos que gentilmente cederam o material para estudo. A T. Camolez pelo auxílio com a bibliografia e identificação dos lagartos. Ao CNPq pela bolsa de iniciação científica (JDFS). A FAPESP pelo auxílio financeiro referente ao processo no. 2011/14080-0 (ASH). Ao D. Verzi e ao revisor anônimo pelos valorosos comentários na revisão do artigo.

\section{REFERÊNCIAS}

Alho, C.J.R. 1982. Brazilian rodents: their habitats and habits. In: M.A. Mares \& H.H. Genoways, (eds.) Mammalian Biology in South America. Pennsylvania: Special Publication, Pymatuning Laboratory of Ecology, University of Pittsburgh, p. 143-166.

Araújo, A.; Rangel, A. \& Ferreira, L.F. 1993. Climatic Change in Northeastern Brazil - Paleoparasitologia Data. Memórias do Institudo Oswaldo Cruz, 88:577-579. doi:org/10.1590/S007402761993000400014

Araújo-Júnior, H.I. \& Porpino, K.O. 2011. Assembleias fossilíferas de mamíferos do Quaternário do Estado do Estado do Rio Grande do Norte, Nordeste do Brasil: diversidade e aspectos tafonômicos e paleoecológicos. Pesquisas em Geociências, 38:67-83.

Arruda, D.A.; De Arruda, J.L.S.; Aued, A.W. \& Cechin, S.T.Z. 2007. 
A dieta de Tropidurus torquatus (Squamata: Tropiduridae) reflete a disponibilidade de presas do meio? In: CONGRESSO DE ECOLOGIA DO BRASIL, 8, 2007. Anais, Caxambu, p. 1-2.

Auler, A.S.; Piló, L.B.; Smart, P.L.; Wang, X.; Hoffmann, D.; Richards, D.A.; Edwards, R.L. Neves, W.A. \& Cheng, H.U. 2006. U-Series dating and taphonomy of Quaternary vertebrates from Brazilian caves. Palaeogeography, Palaeoclimatology, Palaeoecology, 240:508-522. doi:10.1016/j.palaeo.2006.03.002

Ávila-Pires, T.C.S. 1995. Lizards of Brazilian Amazonia (Reptilia: Squamata). Zoologische Verhandelingen, 299:1-706.

Behling, H.; Arz, H.W.; Patzold, J. \& Wefer, G. 2000. Late Quaternary vegetational and climate dynamics in northeastern Brazil, inferences from marine core GeoB 3104-1. Quaternary Science Reviews, 19:981-994. doi:org/10.1016/S0277-3791(99)00046-3

Bezerra, A.M.R. 2008. Revisão taxonômica do gênero Galea Meyen, 1832 (Rodentia, Caviidae, Caviinae). Programa de Pós-Graduação em Biologia Animal, Universidade de Brasília, Tese de Doutorado, 132 p.

Bonvicino, C.R.; Oliveira, J.A. \& D'andrea, P.S. 2008. Guia de Roedores do Brasil, com chaves para gêneros baseadas em caracteres externos. Rio de Janeiro, Centro Pan- Americano de Febre Aftosa, $120 \mathrm{p}$.

Brizuela, S. 2010. Los lagartos continentales fósiles de la Argentina (excepto Iguania). Faculdade de Ciências Naturales y Museo Universidad Nacional de La Plata, Tesis Doctoral, 408 p.

Camolez, T. 2006. Levantamento, identificação e descrição da fauna de Squamata do Quaternário brasileiro (Lepidosauria). Programa de Pós-Graduação em Zoologia, Universidade de São Paulo, Dissertação de Mestrado, 224 p.

Camolez, T. \& Zaher, H. 2010. Levantamento, identificação e descrição da fauna de Squamata do Quaternário brasileiro (Lepidosauria). Arquivos de Zoologia, 41:1-96.

Castro, M.C. \& Langer, M.C. 2011. The mammalian fauna of Abismo Iguatemi, southeastern Brazil. Journal of Cave and Karst Studies, 73:83-92. doi:10.4311/jcks2010pa0140

Contreras, J.R. 1964. Datos acerca de la variación intrapoblacional de la morfología de los molares de entidades de los géneros Galea y Microcavia (Rodentia, Caviidae). Ameghiniana, 3:235-255.

Dantas, M.A.T. 2009. Primeiro registro de fósseis de mamíferos pleistocênicos em caverna de Sergipe, Brasil. Revista Brasileira de Paleontologia, 12:161-164. doi:10.4072/rbp.2009.2.06

De Queiroz, A.N. \& Carvalho, O.A. 2008. Problems in the interpretation of Brazilian archaeofaunas: Different contexts and the important role of taphonomy. Quaternary International, 180:75-89. doi:10.1016/j.quaint.2007.10.024

Eisenberg, J.F. \& Redford, K.H. 1999. Mammals of the NeotropicsThe Central Neotropics: Ecuador, Peru, Bolivia, Brazil. Chicago, University Chicago of Press, 609 p.

Emmons, L.H. 1997. Neotropical rainforest mammals: a field guide. 2.ed. Chicago, University Chicago of Press, 307 p.

Emmons, L.H. 2005. A revision of the gerera of arboreal Echimyidae (Rodentia: Echimyidae, Echimyinae), with descriptions of two new genera. In: E.A. Lacey \& P. Myers (eds.) Mammalian Diversification: from Chromosomes to Phylogeography (a celebration of the Career of James L. Patton). Berkeley, University of California Publications, 133:247-310.

Emmons, L.H.; Leite, Y.L.R.; Kock, D. \& Costa, L.P. 2002. A review of the named forms Phyllomys (Rodentia: Echimyidae) with the description of a new species from coastal Brasil. American Museum Novitates, 3380:1-40.

Emmons, L.H. \& Vucetich, M.G. 1998. The identity of Winge's Lasiuromys villosus and the description of a new genus of
Echimyid Rodent (Rodentia: Echimyidae). American Museum Novitates, 3223:1-12.

Estes, R. 1983. Sauria terrestria, Amphisbaenia. In: P. Wellnhofer (ed.) Handbuch der Paleoherpetologie, Gustav Fisher Verlag, Teil 10A, 249 p.

Estes, R.; De Queiroz, K. \& Gauthier, J. 1988. Phylogenetic relationships within Squamata. In: Estes, R.; Pregill, G. (eds.) Phylogenetic relationships of the lizard families: essays commemorating Charles L. Camp, Stanford University Press, p. 119-281.

Frost, D.R.; Etheridge, R.; Janies, D. \& Titus, T.A. 2001a. Total evidence, sequence alignment, evolution of polychrotid lizards, and a reclassification of the Iguania (Squamata: Iguania). American Museum Novitates, 3343:1-38.

Frost, D.R.; Rodrigues, M.T.; Grant, T. \& Titus, T.A. 2001 b. Phylogenetics of the lizard genus Tropidurus (Squamata: Tropiduridae: Tropidurinae): direct optimization, descriptive efficiency, and sensitivity analysis of congruence between molecular data and morphology. Molecular Phylogenetics and Evolution, 21: 352-371. doi:10.1006/mpev.2001.1015

Geise, L.; Paresque, R.; Sebastião, H.; Shirai, L.T.; Astúa, D. \& Marroig, G. 2010. Non-volant mammals, Parque Nacional do Catimbau, Vale do Catimbau, Buíque, State of Pernambuco, Brazil, with karyologic data. Journal of Species Lists and Distribution, 6:180-186.

Goin, F.J. \& Rey, P. 1997. Sobre las afinidades de Monodelphis Burnett, 1830 (Mammalia: Marsupilia: Didelphidae:Marmosinae). Neotropica, 43:93-98.

Guérin, C. 1991. La faune de vertébrés du Pléistocène supériur de l'aire archéologique de São Raimundo Nonato (Piauí, Brésil). Comptes Rendus de l'Academie dês Sciences. Serie II, Mecanique Physique Chimie Sciences de l'Univers Sciences de La Terre, 312:567-572.

Guérin, C.; Curvello, M.A.; Faure, M.; Hugueney, M. \& MourerChauviré, C. 1993. La faune Pléistocene du Piauí (Nordeste du Brésil): implications paléoécologiques et biochronologiques. Quaternária Nova, 3:303-341.

Hadler, P.; Ferigolo, J. \& Goin, F.J. 2009b. Mamíferos de pequeno porte (Didelphimorphia, Chiroptera e Rodentia) do Pleistoceno final/Holoceno do Brasil, com ênfase no Rio Grande do Sul. In: Ribeiro, A.M.; Bauermann, S.G. \& Scherer, C.S. (Orgs.) Quaternário do Rio Grande do Sul-Integrando Conhecimentos. Porto Alegre, p.155-170 (Monografias da Sociedade Brasileira de Paleontologia).

Hadler, P.; Goin, F.J.; Ferigolo, J. \& Ribeiro, A.M. 2009a. Environmental change and marsupial assemblages in Holocene successions of Southern Brazil. Mammalian Biology, 74:87-99. doi.org/10.1016/j.mambio.2008.03.003

Hadler, P.; Verzi, D.H.; Vucetich, M.G.; Ferigolo, J. \& Ribeiro, A.M. 2008. Caviomorphs (Mammalia, Rodentia) from the Holocene of Rio Grande do Sul State, Brazil: systematics and paleoenvironmental context. Revista Brasileira de Paleontologia, 11:97-116.

Hsiou, A.S. 2007. A new Teiidae specie (Squamata, Scincomorpha) from the Late Pleistocene of Rio Grande do Sul State, Brazil. Revista Brasileira de Paleontologia, 10:181-194.

Hsiou, A.S.; Oliveira, P.V. \& Ximenes, C.L. 2009. Presença de Colubroidea (Squamata, Serpentes) no Quaternário da Província Espeleológica de Ubajara, Estado do Ceará. In: CONGRESSO BRASILEIRO DE PALEONTOLOGIA, 21, 2009. Resumos, Belém, SBP, p. 189.

Huey, R.B. \& Pianka, E.R. 1981. Ecological consequence of foraging mode. Ecology, 62:991-999. 
IBAMA, 2008. Parque Nacional do Catimbau. Accessible at http:// www.ibama.gov.br.

Kerber, L.; Ribeiro, A.M. \& Oliveira, E.V. 2011. The first record of Galea Meyen, 1832 (Rodentia, Hystricognathi, Caviidae) in the late Pleistocene of southern Brazil and its palaeobiogeographic implications. Alcheringa, 29:1-13. doi:10.1080/03115518.20 11.53398

Leite, Y.L.R. 2003. Evolution and systematics of the Atlantic Tree Rats, genus Phyllomys (Rodentia, Echimyidae), with description of two new species. Berkeley, University of California Press, p. 118.

Lessa, G.; Gonçalves, P.R. \& Pessôa, M.L. 2005. Variação geográfica em caracteres cranianos quantitativos de Kerodon rupestris (Wied, 1820) (Rodentia, Caviidae). Arquivos do Museu Nacional, 63:75-88.

Lessa, G. \& Pessôa, L.M. 2005. Variação ontogenética e sexual em caracteres cranianos de Kerodon rupestris Wied, 1820 (Rodentia, Caviidae). Arquivos do Museu Nacional, 63:599-618.

Lôbo, L.R.S.N. 2008. Dinâmica vegetacional e climática holocênica da Caatinga, na região do Parque Nacional do Catimbau, Buique-PE. Programa de Pós-Graduação em Geociências, Universidade Federal de Pernambuco, Dissertação de Mestrado, $137 \mathrm{p}$.

Lund, P.W. 1840. Nouvelles recherches sur la faune fossile Du Brésil (extraits d'une letter addressee aux rédacteurs, et date de Lagoa Santa, ler Avrial 1840). Annales des Sciences Naturelles, Zoologie, 13:310-319.

Martins, M. 1991. The lizards of Balbina, Central Amazonia, Brazil: a qualitative analysis of resource utilization. Studies on Neotropical Fauna and Environment, 26:179-190.

Muniz, S.L.S. \& Santos, E.M. 2009. Répteis do Vale do Catimbau, Buique/PE. In: JORNADA DE ENSINO PESQUISA E EXTENSÃO DA UNIVERSIDADE FEDERAL RURAL DE PERNAMBUCO, 9, 2009. Resumos, Recife, UFRPE, p. 2-4.

Nascimento, A.L. 2001. O sítio Arqueológico Alcobaça: Buíque, Pernambuco - estudo das estruturas arqueológicas. Programa de Pós-Graduação em História, Universidade Federal de Pernambuco, Tese de Doutorado, 186p.

Neves, A.C. da S.A. \& Pessôa, L.M. 2011. Morphological distinction of species of Thrichomys (Rodentia: Echimyidae) through ontogeny of cranial and dental characters. Zootaxa, 2804:15-24.

Nydam, R.L. \& Cifelli, R.L. 2002. A new teiid from the Cedar Mountain Formation (Albian-Cenomanian boundary) of Utah. Journal of Vertebrate Paleontology, 22:276-285. doi:10.1671/0272-4634(2002)022[0276:ANTLFT]2.0.CO;2

Nydam, R.L.; Eaton, J.G. \& Sankey, J. 2007. New taxa of transverselytoothed lizards (Squamata: Scincomorpha) and new information on the evolutionary history of "teiids". Journal of Paleontology, 81:538-549. doi:10.1666/03097.1

Oliveira, E.V.; Barreto, A.M.F. \& Alves, R.S. 2009. Aspectos sistemáticos, paleobiogeográficos e paleoclimáticos dos mamíferos quaternários de Fazenda Nova-PE. Gaea, 5:75-85. doi:10.4013/gaea.2009.52.004

Oliveira, E.V. \& Goin, F.J. 2006. Marsupiais do início do Terciário do Brasil: origem, irradiação e história biogeográfica. In: N.C. Cáceres; E.L.S. Monteiro Filho (eds.) Os marsupiais do Brasil: biologia, conservação e evolução, Editora UFSM, p. 299-320.

Oliveira, J.A.; Gonçalves, P.R. \& Bonvicino, C.R. 2003. Mamíferos da caatinga. In: I.R. Leal; M. Tabarelli; J.M.C. Silva (eds) Ecologia e conservação da Caatinga, Recife, Editora Universitária da UFPE, p. 275-336.

Oliveira, P.V. 2010. Mamíferos do Neopleistoceno-Holoceno do Parque Nacional de Ubajara, Ceará. Programa de PósGraduação em Geociências, Universidade Federal do Rio Grande do Sul, Dissertação de Mestrado, 167 p.

Oliveira, P.V.; Lessa, G.; Viana, M.S.S.; Ximenes, C.L.; Ribeiro, A.M.; Oliveira, E.V.; Santos, A.S.T.; Hsiou, A.S.; Holanda, E.C. \& Kerber, L. 2011. Gruta do urso fóssil (Nordeste do Brasil) e sua Fauna Quaternária: dados preliminares. In: CONGRESSO BRASILEIRO DE PALEONTOLOGIA, 22, 2011. Resumos expandidos, Natal, SBP, p. 651-653.

Perez, C.P. 2009. Paleoecologia de mamíferos viventes como ferramenta na caracterização do ambiente holocênico de Lagoa Santa, MG. Programa de Pós-Graduação em Biociências, Universidade de São Paulo, Dissertação de Mestrado, 112 p.

Presch, W. 1970. The evolution of macroteiid lizards: an osteological interpretation. University of Southern California, $\mathrm{PhD}$. thesis, $255 \mathrm{p}$.

Proença, A.L. 2008. As ocupações pré-coloniais a partir do estudo das paisagens arqueológicas no agreste pernambucano. Programa de Pós-Graduação em História, Universidade Federal de Pernambuco, Dissertação de Mestrado, 97 p.

Ribeiro, L.B. 2010. Ecologia comportamental de Tropidurus hispidus e Tropidurus semitaeniatus (Squamata, Tropiduridae) em simpatria de Caatinga do nordeste do Brasil. Pós-Graduação em Psicobiologia, Universidade Federal do Rio Grande do Norte, PhD. thesis, $79 \mathrm{p}$.

Rodrigues, M.T. 1987. Sistemática, ecologia e zoogeografia dos Tropidurus do grupo torquatus ao sul do Rio Amazonas (Sauria, Iguanidae). Arquivos de Zoologia, 31:105-230.

Rodrigues, P.H. 2008. Didelphimorphia, Chiroptera e Rodentia (Mammalia) do Holoceno do Estado do Rio Grande do Sul, Brasil. Programa de Pós-Graduação em Geociências, Universidade Federal do Rio Grande do Sul, Tese de Doutorado, 203 p.

Salles, L.O.; Cartelle, C.; Guedes, P.G.; Boggiane, P.C.; Janoo, A. \& Russo, C. A.M. 2006. Quaternary mammals from Serra da Bodoquena, Mato Grosso do Sul, Brazil. Boletim do Museu Nacional, Zoologia, 521:1-12.

Silva, F.M.; Alves, R.S.; Barreto. A.M.F.; Sá, F.B. \& Silva, A.C.B.L. 2006. Megafauna Pleistocênica do Estado de Pernambuco. Estudos Geológicos, 16:55-66.

SNE. 2002. Projeto técnico para criação do Parque Nacional do Catimbau/PE. Versão final. Recife, Sociedade Nordestina de Ecologia (SNE), $151 \mathrm{p}$.

Sousa, M.A.N. ; Langguth, A. \& Gimenez, E. 2004. Mamíferos dos Brejos de Altitude de Paraíba e Pernambuco. In: K.C. Porto; Jaime J.P. Cabral \& M. Tabarelli (eds.) Brejos de Altitude em Pernambuco e Paraíba: história natural, ecologia e conservação, Ministério do Meio Ambiente, p. 229-254.

Ubilla, M. 1996. Paleozoologia del Cuaternario continental de la Cuenca Norte del Uruguay: biogeografia, cronologia y aspectos climático-ambientales. 1996. Programa de Pós-Graduação em Zoologia, Universidad de La República, Uruguay, Tesis Doctoral, $232 \mathrm{p}$.

Vanzolini, P.E. 1972. Miscellaneous notes on the ecology of some Brazilian lizards (Sauria). Papéis Avulsos de Zoologia, 26:83115.

Vanzolini, P.E.; Ramos-Costa, A.M.M. \& Vitt, L.J. 1980. Répteis das Caatingas. Rio de Janeiro, Academia Brasileira de Ciências, $161 \mathrm{p}$.

Vital, M.T.A.B.; Santos, F.A.R. \& Alves, M. 2008. Diversidade palinológica das Convolvulaceae do Parque Nacional do Catimbau, Buíque, PE, Brasil. Acta botanica brasilica, 22:11631171. doi:org/10.1590/S0102-33062008000400027 
Vitt, L.J. \& Colli, G.R. 1994. Geographical ecology of a Neotropical lizard: Ameiva ameiva (Teiidae) in Brazil. Canadian Journal of Zoology, 72:1986-2008.

Vitt, L.; Magnusson, W.E.; Ávila-Pires, T.C. \& Lima, A.P. 2008. Guia de Lagartos da Reserva Adolpho Ducke, Amazônia Central. Manaus, Áttema Design Editorial, 176 p.

Wilson, D.E. \& Reeder, D.M. 2005. Mammal species of the World: a taxonomic and geographic reference. Baltimore, The Johns Hopkins University Press, 2142 p.
Winge, H. 1888. Jordfundne og nulevende Gnavare (Rodentia) fra Lagoa Santa, Mias Geraes, Brasilienes e Museo Lundii, med udsigt over gnavernes indbyrdes slægtskab, Copenhagen, $\mathrm{H}$. Hagerup, $178 \mathrm{p}$.

Woods, C.A. \& Howland, E.B. 1979. Adaptive radiation of Capromyid rodents: anatomy of the masticatory apparatus. Journal of Mammalogy, 60:95-116.

Received in March, 2012; accepted in October, 2012. 